\title{
Iron abundance in hot hydrogen-deficient central stars and white dwarfs from FUSE, HST, and IUE spectroscopy ${ }^{\star}$
}

\author{
S. Miksa ${ }^{1,2}$, J. L. Deetjen ${ }^{1}$, S. Dreizler ${ }^{1}$, J. W. Kruk ${ }^{3}$, T. Rauch ${ }^{1}$, and K. Werner ${ }^{1}$ \\ 1 Institut für Astronomie und Astrophysik, Universität Tübingen, Germany \\ 2 Institut für Methodik der Fernerkundung, DLR Oberpfaffenhofen, Germany \\ e-mail: Sabine.Miksa@dlr.de \\ 3 Department of Physics and Astronomy, Johns Hopkins University, Baltimore, MD 21218, USA \\ e-mail: kruk@pha.jhu.edu
}

Received 14 February 2002 / Accepted 25 April 2002

\begin{abstract}
We present a first systematic investigation of the iron abundance in very hot $\left(T_{\text {eff }} \geq 50000 \mathrm{~K}\right)$ hydrogendeficient post-AGB stars. Our sample comprises 16 PG 1159 stars and four DO white dwarfs. We use recent FUSE observations as well as HST and IUE archival data to perform spectral analyses with line blanketed NLTE model atmospheres. Iron is not detected in any PG 1159 star. In most cases this is compatible with a solar iron abundance due to limited quality of HST and IUE data, although the tendency to an iron underabundance may be recognized. However, the absence of iron lines in excellent FUSE spectra suggests an underabundance by at least 1 dex in two objects (K1-16, NGC 7094). A similar result has been reported recently in the [WC]-PG 1159 transition object Abell 78 (Werner et al. 2002). We discuss dust fractionation and s-process neutron-captures as possible origins. We also announce the first identification of sulfur in PG 1159 stars.
\end{abstract}

Key words. stars: AGB and post-AGB - stars: atmospheres - stars: abundances - stars: evolution - white dwarfs ultraviolet: stars

\section{Introduction}

PG 1159 stars are hot $\left(T_{\text {eff }}=65000-180000 \mathrm{~K}\right)$ hydrogendeficient post-AGB stars with carbon, helium and oxygen as main atmospheric constituents. They are thought to be the result of a late helium shell flash (see e.g. Werner 2001). Since they belong to the disk population and since diffusion effects can be excluded due to ongoing mass-loss, one expects solar iron abundances.

Up to now no iron abundances were determined for these objects, for two reasons. First, iron is highly ionized so that spectral lines have to be looked for in the UV and FUV region. High resolution, high- $S / N$ spectra are required from these mostly faint objects. A few of the brightest were observed with IUE, however, line identification is doubtful or impossible, as we will show. HST spectra of many PG 1159 stars are archived; however, in most cases the spectral resolution is not high. In this paper we use all this archival material to make a systematic investigation of iron lines with NLTE model atmospheres. We also use new

Send offprint requests to: K. Werner, e-mail: werner@astro.uni-tuebingen.de

* Based on observations obtained with the Far Ultraviolet Spectroscopic Explorer (FUSE), the Hubble Space Telescope (HST), and the International Ultraviolet Explorer (IUE).
FUSE spectra of seven PG 1159 stars and of the hottest known DO white dwarf for this purpose. Second, spectral analyses require NLTE model atmospheres. Models that include iron opacities for high ionization stages have only recently become available.

\section{Sample selection and observational data}

Our sample comprises all PG 1159 stars for which HST and IUE high resolution archival spectra with a minimum acceptable $S / N$ are available. These are 16 out of the 35 objects known. FUSE spectra are available for seven of the program stars. The sample is augmented by four hot non-DA (DO) white dwarfs in order to complete an earlier analysis of DO spectra (Dreizler 1999). The twenty program stars are listed in Table 1 . The spectra used for our analysis are listed in Table 2.

\subsection{FUSE}

The FUSE datasets in programs P132 and Q109 were observed for the present study of PG 1159 stars, the datasets in program P104 were observed for ISM studies, and the datasets in program M107 were observed for the FUSE calibration program. Each of these datasets is available from 
Table 1. Atmospheric parameters of the program stars as taken from the literature. The iron abundance is determined in this work, except for Abell 78. Abundances are given in \% number fractions, except for iron. The iron abundance in PG 1159 stars is the logarithm of mass fraction relative to the solar value. For the DO stars Fe is given as number ratio relative to He. Pulsating PG 1159 stars are marked with an asterisk.

\begin{tabular}{|c|c|c|c|c|c|c|c|c|c|c|}
\hline Object & type & $\begin{array}{r}T_{\text {eff }} \\
{[\mathrm{kK}]}\end{array}$ & $\begin{array}{l}\log g \\
(\operatorname{cgs})\end{array}$ & $\mathrm{H}$ & $\mathrm{He}$ & $\mathrm{C}$ & $\mathrm{N}$ & $\mathrm{O}$ & $\begin{array}{l}\log \mathrm{Fe} / \mathrm{Fe}_{\odot} \\
\text { (mass fraction) }\end{array}$ & ref. \\
\hline RX J $2117.1+3412$ & PG 1159* & 170 & 6.0 & & 61 & 31 & & 8 & $\leq 0$ & $\mathrm{~A}$ \\
\hline PG $1520+525$ & PG 1159 & 150 & 7.5 & & 72 & 21 & $<0.01$ & 7 & $<-0.5$ & $\mathrm{~L}$ \\
\hline NGC 246 & PG 1159* & 150 & 5.7 & & 85 & 13 & & 2 & $\leq 0$ & $\mathrm{H}$ \\
\hline PG 1159-035 & PG $1159 *$ & 140 & 7.0 & $<5$ & 61 & 30 & 1 & 8 & $<-0.5$ & $\mathrm{~K}, \mathrm{~L}$ \\
\hline K 1-16 & PG 1159* & 140 & 6.4 & & 61 & 31 & & 8 & $<-1.0$ & I \\
\hline HS $2324+397$ & PG $1159 *$ & 130 & 6.2 & 61 & 30 & 9 & $<0.1$ & 0.2 & $<-0.5$ & $\mathrm{D}$ \\
\hline Abell 43 & PG $1159 *$ & 110 & 5.7 & 74 & 22 & 4 & & & $\leq 0$ & $\mathrm{G}$ \\
\hline Abell 78 & PG 1159 & 110 & 5.5 & & 62 & 31 & & 7 & $<-1.5$ & $\mathrm{~J}, \mathrm{M}$ \\
\hline NGC 7094 & PG $1159 *$ & 110 & 5.7 & 74 & 22 & 4 & & & $<-1.5$ & $\mathrm{G}$ \\
\hline PG $1424+535$ & PG 1159 & 110 & 7.0 & & 76 & 22 & $<0.001$ & 2 & $\leq 0$ & $\mathrm{~L}$ \\
\hline HS $1517+7403$ & PG 1159 & 110 & 7.0 & & 95 & 5 & $<0.001$ & 0.5 & $\leq 0$ & $\mathrm{~L}$ \\
\hline PG 2131+066 & PG $1159 *$ & 95 & 7.5 & & 71 & 21 & 1 & 7 & $\leq 0$ & $\mathrm{~L}$ \\
\hline MCT 0130-1937 & PG 1159 & 90 & 7.5 & & 90 & 9 & $<0.001$ & 1 & $\leq 0$ & $\mathrm{~L}$ \\
\hline PG $1707+427$ & PG 1159* & 85 & 7.5 & & 71 & 21 & 1 & 7 & $\leq 0$ & $\mathrm{~L}$ \\
\hline PG $0122+200$ & PG $1159 *$ & 80 & 7.5 & & 71 & 21 & 1 & 7 & $\leq 0$ & $\mathrm{~L}$ \\
\hline \multirow[t]{2}{*}{ HS $0704+6153$} & PG 1159 & 75 & 7.0 & & 88 & 9 & $<0.001$ & 3 & $\leq 0$ & $\mathrm{~L}$ \\
\hline & & & & & & & & & $\begin{array}{l}\mathrm{Fe} / \mathrm{He} \\
\text { (number ratio) }\end{array}$ & \\
\hline KPD $0005+5106$ & $\mathrm{DO}$ & 120 & 7.0 & & 100 & 0.1 & 0.01 & $<10^{-5}$ & $<10^{-5}$ & $\mathrm{~A}$ \\
\hline PG $1034+001$ & $\mathrm{DO}$ & 100 & 7.5 & $<5$ & 100 & 0.001 & 0.063 & 0.0079 & $1 \times 10^{-5}$ & $\mathrm{~B}, \mathrm{C}$ \\
\hline HZ 21 & DO & 53 & 7.8 & $<10$ & 100 & $<0.001$ & $<0.001$ & $<0.001$ & $<10^{-4}$ & $\mathrm{E}$ \\
\hline HD 149499B & DO & 50 & 8.0 & 18 & 82 & 0.001 & & & $<10^{-5}$ & $\mathrm{E}, \mathrm{F}$ \\
\hline
\end{tabular}

References in last column: A: Werner et al. (1996), B: Werner (1996), C: Werner et al. (1995), D: Dreizler et al. (1996), E: Dreizler \& Werner (1996), F: Napiwotzki et al. (1995), G: Dreizler et al. (1997), H: Rauch \& Werner (1997), I: Kruk \& Werner (1998), J: Werner \& Koesterke (1992), K: Werner et al. (1991), L: Dreizler \& Heber (1998), M: Werner et al. (2002).

the Multimission Archive at the Space Telescope Science Institute (MAST). All observations were obtained using the $30^{\prime \prime} \times 30^{\prime \prime}$ LWRS spectrograph aperture. The data were obtained in "timetag" mode, except for P1320501 and M1070201,2,3 datasets, which were obtained in "histogram" (or spectral image) mode. These datasets were all reduced using the standard CALFUSE pipeline, version 1.8.7. These observations exhibit RMS variations in observed flux that are usually less than $1 \%$ from one exposure to another, indicating that no significant signal was lost as a result of channel misalignments. The one exception was $\mathrm{SiC} 1$ data for exposure 1 of the observation of RXJ $2117.1+3412$, for which the flux level was about $20 \%$ below that of the other exposures. A description of the FUSE instrument and the channel alignment issues are given by Moos et al. (2000) and Sahnow et al. (2000). For most objects the spectra obtained in different exposures were coaligned by cross-correlating on regions containing narrow interstellar absorption features before combining to produce a spectrum for an entire observation. The resulting spectral resolution was typically about $20 \mathrm{~km} \mathrm{~s}^{-1}$. The spectra obtained in single exposures for the objects HS $2324+397$ and PG $1707+427$ did not have sufficient signal-to-noise ratios to permit cross-correlation, so these spectra were combined without coalignment. In these cases the spectral resolution was somewhat worse, about $25 \mathrm{~km} \mathrm{~s}^{-1}$ for PG $1707+427$, and $25-30 \mathrm{~km} \mathrm{~s}^{-1}$ for HS $2324+397$.

The observation of K 1-16 required special processing. This observation was obtained during in-orbit checkout very early in the mission, as part of a test to determine both the channel alignment offsets and the primary mirror to spectrograph slit focus offset for each channel. The test consisted of slewing the telescope in $401^{\prime \prime}$ steps during each exposure, centered on the LiF1 LWRS aperture. There were a total of 11 scans each in the dispersion and cross-dispersion directions. Because the channels were only crudely aligned at the time of this test, the effective exposure time varied for each channel, from about $38 \mathrm{ksec}$ for LiF1 to only $8 \mathrm{ksec}$ for $\mathrm{SiC} 2$. Data were discarded for time periods in which the star was outside of the aperture, or for which the count rate otherwise deviated significantly from the mean. These data were processed using version 1.7.7 of CALFUSE, but the differences between this version and 1.8.7 were not significant for the purposes of this program. Custom processing was added to the pipeline to correct the recorded photon positions for the slewing of the spacecraft across the spectrograph 
Table 2. List of spectra for the program stars. The IUE spectra cover a wavelength range of $1150-1980 \AA$ with a resolution of about $0.1 \AA$. The available FUSE spectra cover $905-1187 \AA$ with a resolution of about $0.05 \AA$.

\begin{tabular}{|c|c|c|c|c|c|c|c|c|}
\hline \multirow[t]{2}{*}{ Object } & \multicolumn{4}{|c|}{ - HST - } & \multicolumn{2}{|c|}{ - IUE - } & \multicolumn{2}{|c|}{ - FUSE - } \\
\hline & $\begin{array}{l}\text { dataset } \\
\text { name }\end{array}$ & $\begin{array}{c}\lambda \text {-range } \\
{[\AA]}\end{array}$ & $\begin{array}{l}\text { res. } \\
{[\AA]}\end{array}$ & $\begin{array}{c}t_{\exp } \\
{[\mathrm{min}]}\end{array}$ & $\begin{array}{l}\text { SWP } \\
\text { number }\end{array}$ & $\begin{array}{c}t_{\exp } \\
{[\mathrm{min}]}\end{array}$ & $\begin{array}{l}\text { dataset } \\
\text { name }\end{array}$ & $\begin{array}{c}t_{\exp } \\
{[\min ]}\end{array}$ \\
\hline \multirow[t]{3}{*}{ RX J 2117.1+3412 } & Z27J0206T & $1227-1263$ & 0.06 & 13 & 47556 & 360 & P1320501 & 137 \\
\hline & Z27J0207N & 1273-1309 & 0.06 & 11 & 47563 & 362 & & \\
\hline & Z27J0208T & $1343-1379$ & 0.06 & 16 & 55411 & 363 & & \\
\hline \multirow{10}{*}{$\begin{array}{l}\text { PG } 1520+525 \\
\text { NGC } 246\end{array}$} & $\mathrm{Z} 2 \mathrm{~T} 20104 \mathrm{~T}$ & $1165-1461$ & 0.60 & 24 & & & P1320101 & 81 \\
\hline & & & & & 03353 & 112 & & \\
\hline & & & & & 41997 & 120 & & \\
\hline & & & & & 42068 & 223 & & \\
\hline & & & & & 42073 & 115 & & \\
\hline & & & & & 42104 & 150 & & \\
\hline & & & & & 42214 & 150 & & \\
\hline & & & & & 42247 & 120 & & \\
\hline & & & & & 47843 & 165 & & \\
\hline & & & & & 47844 & 155 & & \\
\hline \multirow[t]{4}{*}{ PG 1159-035 } & & & & & 23032 & 1020 & Q1090101 & 105 \\
\hline & & & & & 53903 & 667 & & \\
\hline & & & & & 54675 & 875 & & \\
\hline & & & & & 54976 & 880 & & \\
\hline \multirow[t]{2}{*}{ K $1-16$} & Z1EJ0304M & $1497-1532$ & 0.06 & 13 & & & I8110302 & 665 \\
\hline & Z1EJ0305M & $1525-1560$ & 0.06 & 13 & & & & \\
\hline HS $2324+397$ & Z3GW0204T & $1140-1436$ & 0.60 & 74 & & & P1320601 & 67 \\
\hline Abell 43 & Z3GW0304T & $1139-1435$ & 0.60 & 71 & 38955 & 385 & & \\
\hline \multirow[t]{3}{*}{ Abell 78} & & & & & 16967 & 40 & & \\
\hline & & & & & 19879 & 425 & & \\
\hline & & & & & 19906 & 420 & & \\
\hline \multirow[t]{4}{*}{ NGC 7094} & Z3GW0104T & $1139-1435$ & 0.60 & 22 & 52919 & 330 & P1043701 & 386 \\
\hline & Z3GW0105T & $1455-1751$ & 0.60 & 47 & 56107 & 400 & & \\
\hline & & & & & 56112 & 400 & & \\
\hline & & & & & 56120 & 400 & & \\
\hline PG $1424+535$ & Z2T20204T & $1166-1452$ & 0.60 & 24 & & & & \\
\hline HS $1517+7403$ & Z2T20704T & $1165-1451$ & 0.60 & 24 & & & & \\
\hline PG $2131+066$ & Z2T20804T & $1165-1451$ & 0.60 & 18 & & & & \\
\hline MCT 0130-1937 & Z2T20504T & $1165-1451$ & 0.60 & 17 & & & & \\
\hline PG $1707+427$ & Z2T20304T & $1165-1451$ & 0.60 & 20 & & & P1320401 & 243 \\
\hline PG $0122+200$ & $\mathrm{Z} 2 \mathrm{~T} 20404 \mathrm{~T}$ & $1164-1450$ & 0.60 & 18 & & & & \\
\hline \multirow{2}{*}{ HS $0704+6153$} & Z2T20604T & $1165-1461$ & 0.60 & 27 & & & & \\
\hline & Z2T20605T & $1165-1461$ & 0.60 & 27 & & & & \\
\hline \multirow[t]{4}{*}{ KPD 0005+5106 } & Z27J0106T & $1227-1263$ & 0.06 & 13 & 26191 & 420 & M1070201 & 132 \\
\hline & Z27J0107T & $1273-1309$ & 0.06 & 11 & 52108 & 360 & M1070202 & 77 \\
\hline & Z27J0108T & $1343-1379$ & 0.06 & 16 & 52146 & 425 & M1070203 & 138 \\
\hline & & & & & 52185 & 425 & P1040101 & 114 \\
\hline \multirow[t]{5}{*}{ PG $1034+001$} & Z0YE0C08T & $1185-1221$ & 0.06 & 5 & 18509 & 330 & & \\
\hline & Z0YE0C09T & $1221-1256$ & 0.06 & 10 & 26201 & 262 & & \\
\hline & Z0YE0C0AT & $1369-1406$ & 0.06 & 5 & & & & \\
\hline & Z0YE0C0CT & $1532-1568$ & 0.06 & 5 & & & & \\
\hline & Z0YE0C0DT & $1622-1658$ & 0.06 & 5 & & & & \\
\hline \multirow[t]{2}{*}{ HZ 21} & Z3GM0404T & $1227-1265$ & 0.06 & 42 & 31287 & 1147 & & \\
\hline & Z3GM0405T & $1339-1375$ & 0.06 & 33 & & & & \\
\hline \multirow[t]{5}{*}{ HD 149499B } & Z3GM0504P & $1227-1265$ & 0.06 & 7 & 06272 & 90 & & \\
\hline & Z3GM0505P & $1338-1375$ & 0.06 & 7 & 13781 & 40 & & \\
\hline & Z3GM0506P & $1699-1735$ & 0.06 & 9 & 13782 & 28 & & \\
\hline & & & & & 13783 & 89 & & \\
\hline & & & & & 17467 & 75 & & \\
\hline
\end{tabular}




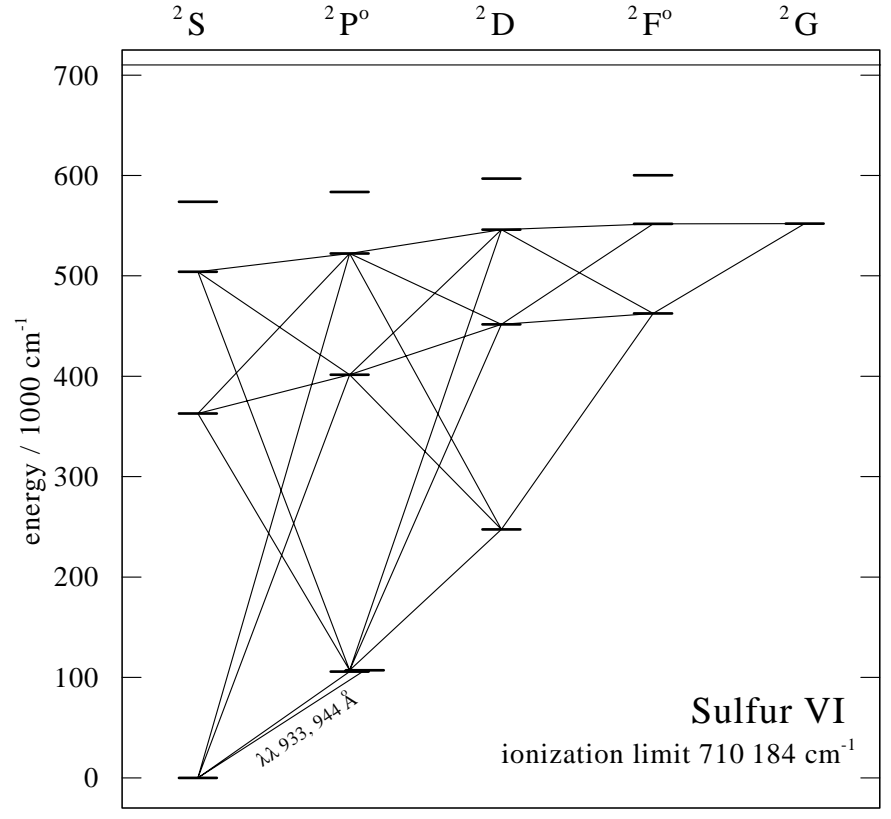

Fig. 1. Model atom for S VI. The transition responsible for the $\mathrm{S}$ VI resonance doublet in the FUSE range is labeled.

apertures during each exposure. The resulting resolution was about $24 \mathrm{~km} \mathrm{~s}^{-1}$.

\subsection{HST and IUE}

HST data was retrieved from the MAST. The spectra were taken with the Goddard High Resolution Spectrograph (GHRS) with a resolution of $0.06 \AA$ or $0.6 \AA$. All exposures were made in the FPSPLIT mode which results in four individual spectra. As only the first exposure was wavelength-calibrated, the subsequent exposures were adjusted in wavelength to match the first using crosscorrelation.

Reduced SWP spectra were extracted from the IUE Final Archive using preview data. They cover a wavelength range of $1150-1980 \AA$, with a resolution of about $0.1 \AA$. If more than one spectrum was available they were co-added using the $S / N$-ratio as weighting factor.

\section{Model atmospheres and synthetic spectra}

We have calculated a plane-parallel model atmosphere in radiative and hydrostatic equilibrium for each program star with our ALI code (Werner \& Dreizler 1999) which can handle line blanketing by the iron group elements. We use the line list of Kurucz (1991). In general the models are similar to those of Deetjen et al. (1999), so we will restrict ourselves to a summary of the model atoms in Table 3 . However, higher ionization stages of iron are taken into account due to the higher effective temperature of our program stars.

Sulfur line formation calculations were performed for the central star of K1-16. The sulfur model atom (S IV-VII) has been constructed using the Opacity Project

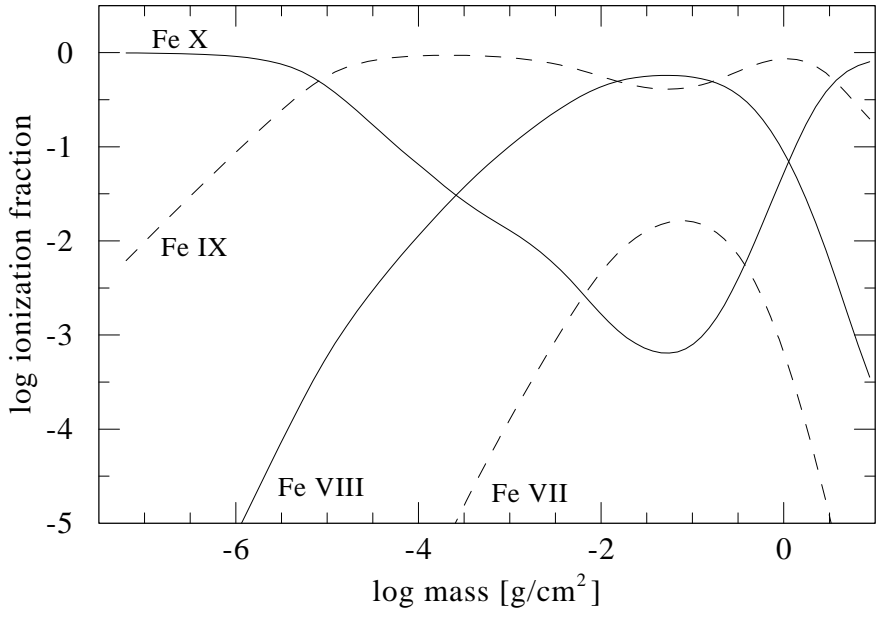

Fig. 2. Ionization structure of the model atmosphere for K 1-16 with $T_{\text {eff }}=140000 \mathrm{~K}, \log g=6.4$ and a solar iron abundance.

Table 3. Summary of the model atoms used in the model atmosphere calculations. The numbers in brackets give the individual line numbers summed into superlines for the iron ions. Each model atom is closed by a single NLTE level representing the highest ionization stage. These levels are not specified here.

\begin{tabular}{llrrr}
\hline \hline element & ion & NLTE levels & lines & \\
\hline H & I & 5 & 6 & \\
He & I & 1 & 0 & \\
& II & 32 & 79 & \\
O & IV & 1 & 0 & \\
& V & 6 & 0 & \\
& VI & 36 & 102 & \\
& VII & 1 & 0 & \\
N & IV & 6 & 4 & \\
& V & 4 & 1 & \\
& VI & 1 & 0 & \\
C & III & 1 & 0 & \\
& IV & 36 & 96 & \\
& V & 1 & 0 & \\
Fe & III & 7 & 25 & $(301981)$ \\
& IV & 7 & 25 & $(1027793)$ \\
& V & 7 & 25 & $(793718)$ \\
& VI & 8 & 33 & $(340132)$ \\
& VII & 9 & 39 & $(86504)$ \\
& VIII & 7 & 27 & $(8724)$ \\
& IX & 8 & 32 & $(36843)$ \\
\hline \multirow{2}{*}{ total } & & 184 & 494 & $(2595695)$ \\
\hline & & & & \\
\hline
\end{tabular}

(OP, Seaton et al. 1994) database which is a basic source for energy levels, oscillator strengths, and photoionization cross-sections. Since the OP energy levels are slightly different from laboratory measurements, we have replaced these values by those listed in Bashkin \& Stoner (1975). The Grotrian diagram in Fig. 1 shows the levels and line transitions of the S VI model ion. 

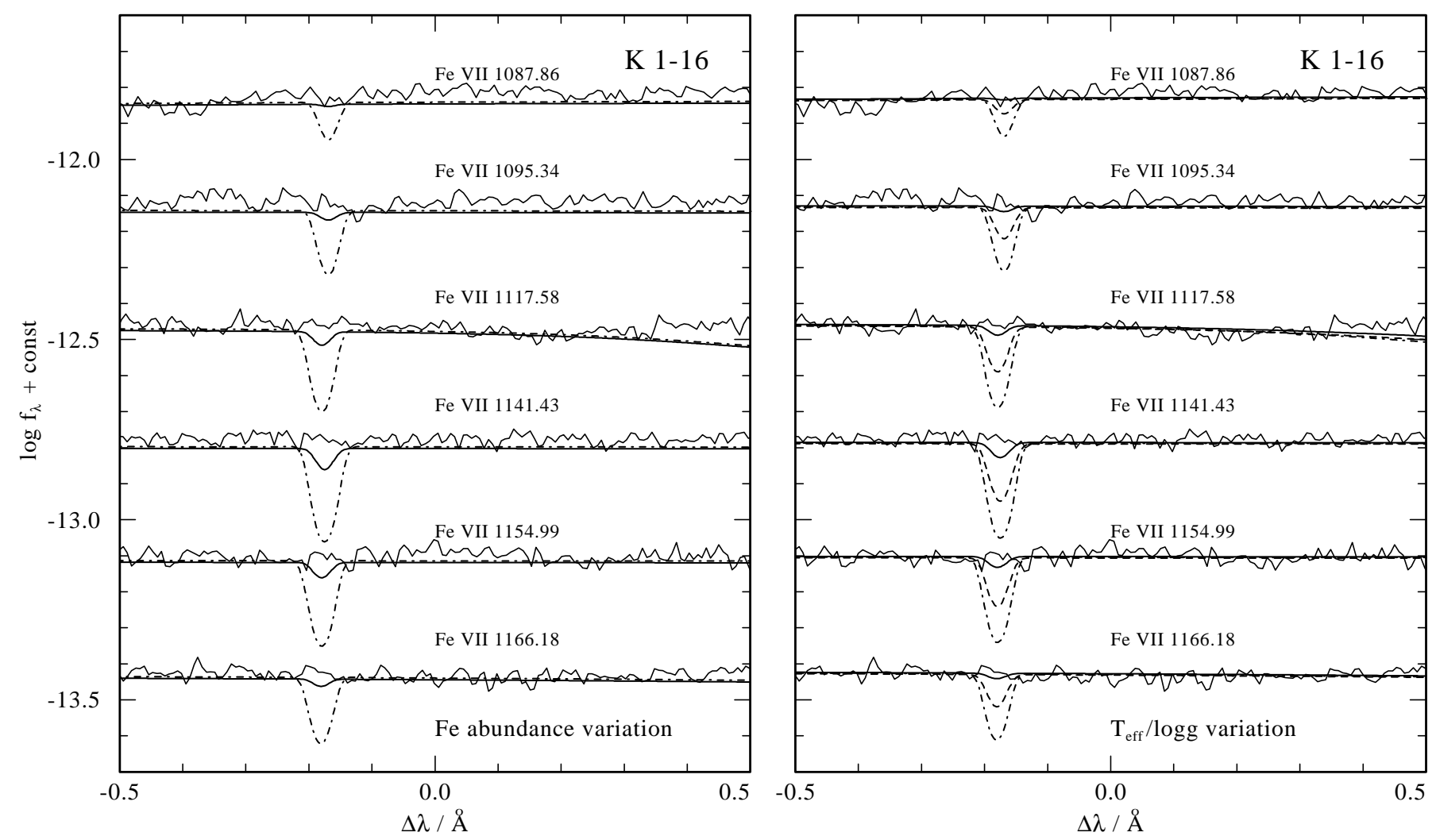

Fig. 3. The strongest iron lines expected in the FUSE data of K 1-16 are from Fe VII. None of these can be identified in K 1-16 (LiF2A data). Left panel: models with $T_{\text {eff }}=140000 \mathrm{~K}, \log g=6.4$ and iron abundances of 0.1 solar (solid) and solar (dashdotted line). Right panel: models with $T_{\text {eff }}=140000 \mathrm{~K} / \log g=6.4$ (dash-dotted), 150 000/6.5 (dashed) and 160 000/6.6 (solid), and solar iron abundance. A velocity shift of $-48.07 \mathrm{~km} \mathrm{~s}^{-1}$ is taken into account according to Holberg et al. (1998). The spectra are smoothed with $0.01 \AA$ Gaussians.

\section{Iron abundance}

For most of the objects the quality of the spectroscopic data is not sufficient to detect iron lines when compared with models with solar Fe abundance (all abundance values are given in mass fractions unless otherwise noted). For these objects we can only conclude that the iron abundance is less or equal solar (Table 1).

\subsection{PG 1159 stars}

Before we discuss individual objects, we briefly go into those stars for which a solar abundance of Fe cannot be strictly excluded.

For PG $1707+427$ the FUSE data is superior to the HST data, however, the $S / N$ of the FUSE spectrum is still too poor to detect potential Fe VI and Fe VII lines. For PG 1424+535, HS 1517+7403, PG 2131+066, MCT 01301937, PG $0122+200$, and HS $0704+6153$ only HST data exist, but their resolution $(0.6 \AA)$ is too low. For Abell 43 IUE and HST spectra exist, but with insufficient $S / N$ and resolution, respectively. Hence, for all the objects mentioned in this paragraph, Fe lines remain hidden in the spectra if the abundance is solar.

In contrast to our model predictions no Fe vi or Fe VI/VII lines can be detected in the FUSE spectra of
HS $2324+397$ and PG $1520+525$, respectively. We derive a slight underabundance of 0.5 dex, but due to the relatively low $S / N$ we must accept that a solar Fe abundance cannot be excluded rigorously. The HST data are not useful because of insufficient resolution $(0.6 \AA)$.

For PG 1159-035 four co-added IUE spectra were analyzed, with no clear detection of potential Fe viI lines. Also, Fe VII lines are not detectable in the FUSE spectra, hence, $\mathrm{Fe}$ is possibly underabundant by 0.5 dex or more.

\subsubsection{K $1-16$}

K 1-16 is an extremely hot PG 1159 type central star. Due to its high effective temperature $(140000 \mathrm{~K})$ the principal ionization stages of iron in the line formation regions of the atmosphere are Fe VIII and Fe IX (Fig. 2). A comparison of the excellent FUSE data with model spectra reveals, that Fe VII lines should be easily detectable if the effective temperature is indeed $140000 \mathrm{~K}$ (Rauch \& Werner 1997) and if the Fe abundance is solar. Figure 3, however, shows that the strongest Fe VII model lines cannot be detected in the FUSE spectrum. Varying $T_{\text {eff }}$ and Fe abundance in the model suggests that either the Fe abundance is lower than one tenth solar or that the effective temperature is higher than $160000 \mathrm{~K}$. The high $T_{\text {eff }}$ would be surprising, because it means that the quoted value, which was 
determined from optical spectra, would be in error by at least $20000 \mathrm{~K}$. This is larger than a typical error of $10 \%$ for such analyses, however, the high $T_{\text {eff }}$ cannot be excluded definitely. Hence we conclude that an Fe underabundance of at least one dex is probably causing the failure to detect Fe VII lines in the FUSE data. Another argument for the Fe underabundance can be given. Two Fe Ix lines with relatively large $g f$-values are known, which are located in the FUSE spectral region, contrary to most other lines of this ion, which are found in the $100-600 \AA$ region. These lines (at $955.84 \AA$ and $973.71 \AA$ ) clearly show up in the models with solar Fe abundance, but nothing is seen in the FUSE spectrum at these wavelengths (Fig. 4). This holds particularly for the hotter model $(160000 \mathrm{~K})$, corroborating an underabundance even in the case that $T_{\text {eff }}$ is higher than previously found.

A final decision about the possibility of an Fe underabundance in $\mathrm{K}$ 1-16 would require a high-precision analysis of He, C, O line profiles to fix $T_{\text {eff }}$ as accurately as possible, using improved optical and UV spectroscopy in combination with the FUSE data. Alternatively, the search for the bulk of strongest Fe VIII and Fe IX lines in the 100-200 $\AA$ range could be feasible with the Chandra observatory because $\mathrm{K} 1-16$ is a soft X-ray source detected by ROSAT and EUVE.

The lack of Fe vir lines in the K 1-16 FUSE data supports a result by Holberg et al. (1998). They found no evidence of Fe VII lines in (much less meaningful) IUE spectra, contrary to earlier work by Feibelman \& Bruhweiler (1990).

\subsubsection{RX J $2117.1+3412$}

RX J 2117.1+3412 is the hottest known PG 1159-type central star $\left(T_{\text {eff }}=170000 \mathrm{~K}\right)$. From the discussion above it is clear, that the iron ionization is so high that Fe VII lines are not detectable, even in excellent data. This is indeed true when inspecting the FUSE spectra. It is interesting to note that earlier identifications of the Fe VII $1239.69 \AA$ line in a high resolution HST/GHRS spectrum (Werner et al. 1996) and in IUE spectra (Feibelman 1999) are therefore most likely wrong. Our model predicts that none of the potential Fe VII lines in the GHRS and IUE range appears. We believe that the observed absorption is in fact an interstellar feature. There are two $\mathrm{Mg}$ II lines nearby, at $1239.93 \AA$ and $1240.39 \AA$. The redshift of the photospheric spectrum $\left(+47.22 \mathrm{~km} \mathrm{~s}^{-1}\right.$ from Holberg et al. 1998, confirmed by our measurement of the C IV $1107.93 \AA$ line) and the blueshift of the ISM lines $\left(-2.39 \mathrm{~km} \mathrm{~s}^{-1}\right)$ conspire in a way that the interstellar Mg II $1239.93 \AA$ line almost coincides with the position of a possible stellar Fe VII line. The same problem arises in the case of KPD 0005+5106 (see below). Generally, care must therefore be taken when iron is identified in hot stars by means of this Fe VII $1239.69 \AA$ line alone. This can only be regarded as safe when confusion with interstellar $\mathrm{Mg}$ II can be excluded. The two Fe Ix lines introduced above cannot

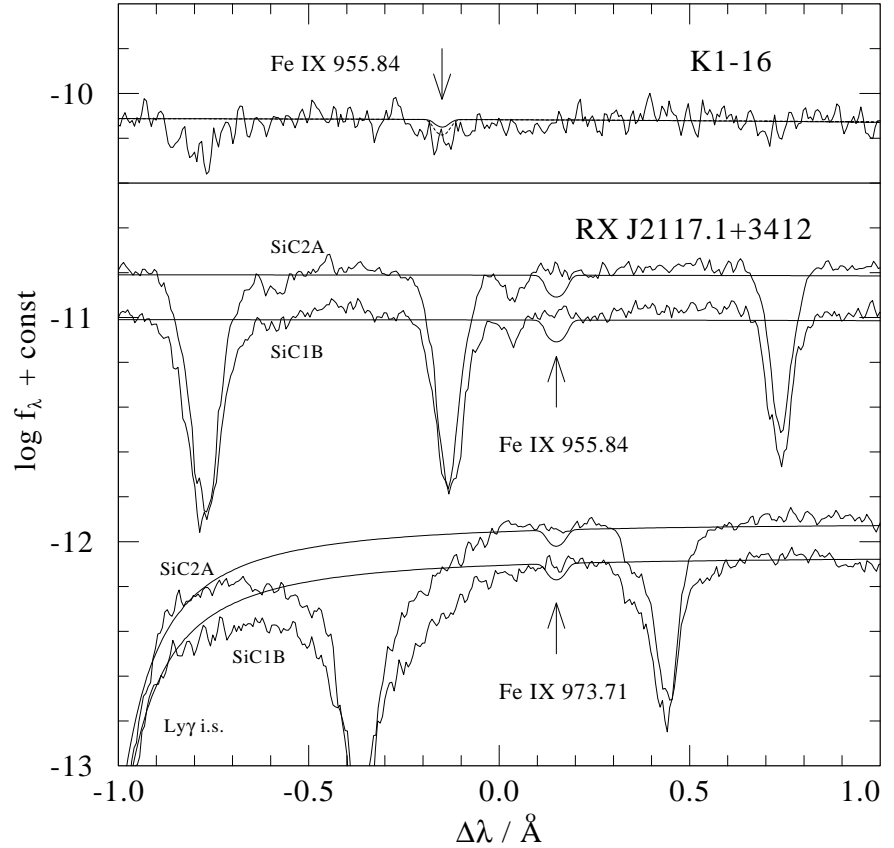

Fig. 4. Two Fe IX lines are located in the FUSE range. Top panel: K 1-16 compared to two model spectra $\left(T_{\text {eff }}=140000 \mathrm{~K}\right.$, $\log g=6.4$, full; $T_{\text {eff }}=160000 \mathrm{~K}, \log g=6.6$, dashed; solar Fe abundance) centered near the Fe IX $956 \AA$ line. Bottom panel: RX J 2117.1+3412 compared to the model with parameters as in Table 1 ( $T_{\text {eff }}=170000 \mathrm{~K}, \log g=6.0$, solar Fe abundance) centered around Fe IX $956 \AA$ and Fe IX $974 \AA$. For this star both detector segments ( $\mathrm{SiC} 2 \mathrm{~A}$ and $\mathrm{SiC} 1 \mathrm{~B}$, slightly shifted vertically) are shown. The spectra are smoothed with $0.01 \AA$ Gaussians. A radial velocity shift of $+47.22 \mathrm{~km} \mathrm{~s}^{-1}$ is taken into account.

be identified in the FUSE spectrum (Fig. 4). Like in the case of K1-16 this could hint at an Fe underabundance and soft X-ray spectroscopy is required for a quantitative analysis.

\subsubsection{NGC 246}

NGC 246 is also a very hot $\left(T_{\text {eff }}=150000 \mathrm{~K}\right)$ PG 1159 type central star. Numerous IUE high resolution spectra exist which can be co-added. Holberg et al. (1998) find no evidence of the Fe viI lines reported by Feibelman \& Bruhweiler (1990). We confirm this result and find that this is due to the high temperature. Better spectra (from HST or FUSE) might be able to detect these lines, which are weak but not completely absent in the solar Fe abundance models. It appears that the tentative identification of lines from even lower ionized Fe vi by Feibelman \& Johansson (1995) and Feibelman (1995) is very likely not correct.

\subsubsection{NGC 7094}

NGC 7094 is a so-called hybrid PG 1159 star, meaning that detectable amounts of hydrogen are present in its atmosphere. We fail to detect iron lines in the IUE and 


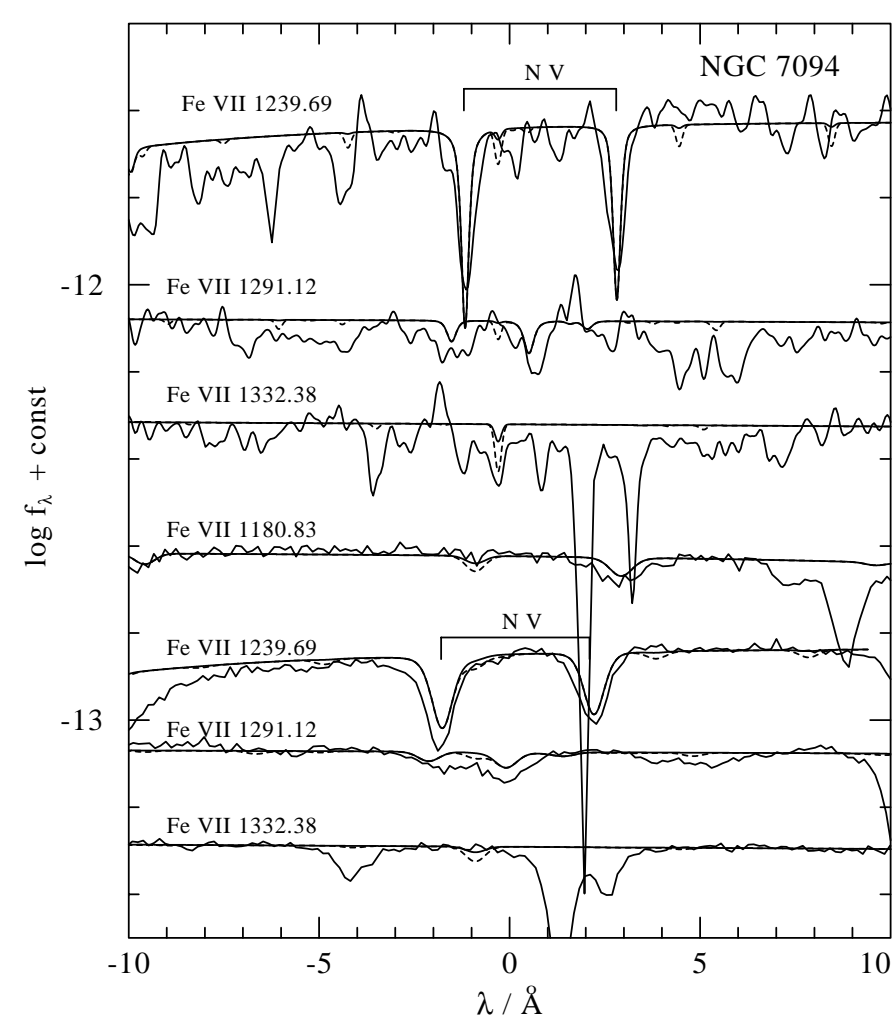

Fig. 5. The strongest Fe VII lines in the spectra of NGC 7094. The top three lines are fitted to IUE spectra, the four lines at the bottom are fitted to HST spectra. The models have iron abundances of 0.1 solar (solid line) and solar (dashed line). No iron lines can be identified. The IUE and HST spectra are smoothed $0.2 \AA$ and $0.6 \AA F W H M$ Gaussians, respectively. The calculated spectra were shifted accounting for radial velocity. Note that the radial velocity of the photospheric lines from HST data differs from that of the IUE data.

HST spectra of NGC 7094 (Fig. 5). This is in contrast to Feibelman (2000), who claimed identification of several Fe VI and Fe VII lines in the IUE data. This central star is so hot that our model predicts stronger Fe VII than Fe VI lines. One can derive an underabundance of 0.5 dex. Inspecting the superior FUSE data we, again, cannot identify Fe viI lines. Comparison with our model reveals an even higher underabundance of 1-2 dex (Fig. 6).

\subsubsection{Abell 78}

The central star of the planetary nebula Abell 78 is one of the rare [WC]-PG 1159 transition object showing spectral signatures of both early [WC] spectral type (emission lines) and PG 1159 type (absorption lines). As in the case of NGC 7094 only the FUSE spectrum allows one to determine a strict limit to the Fe abundance. A first analysis has been presented recently by Werner et al. (2002) and the result is an underabundance of $1-2$ dex.

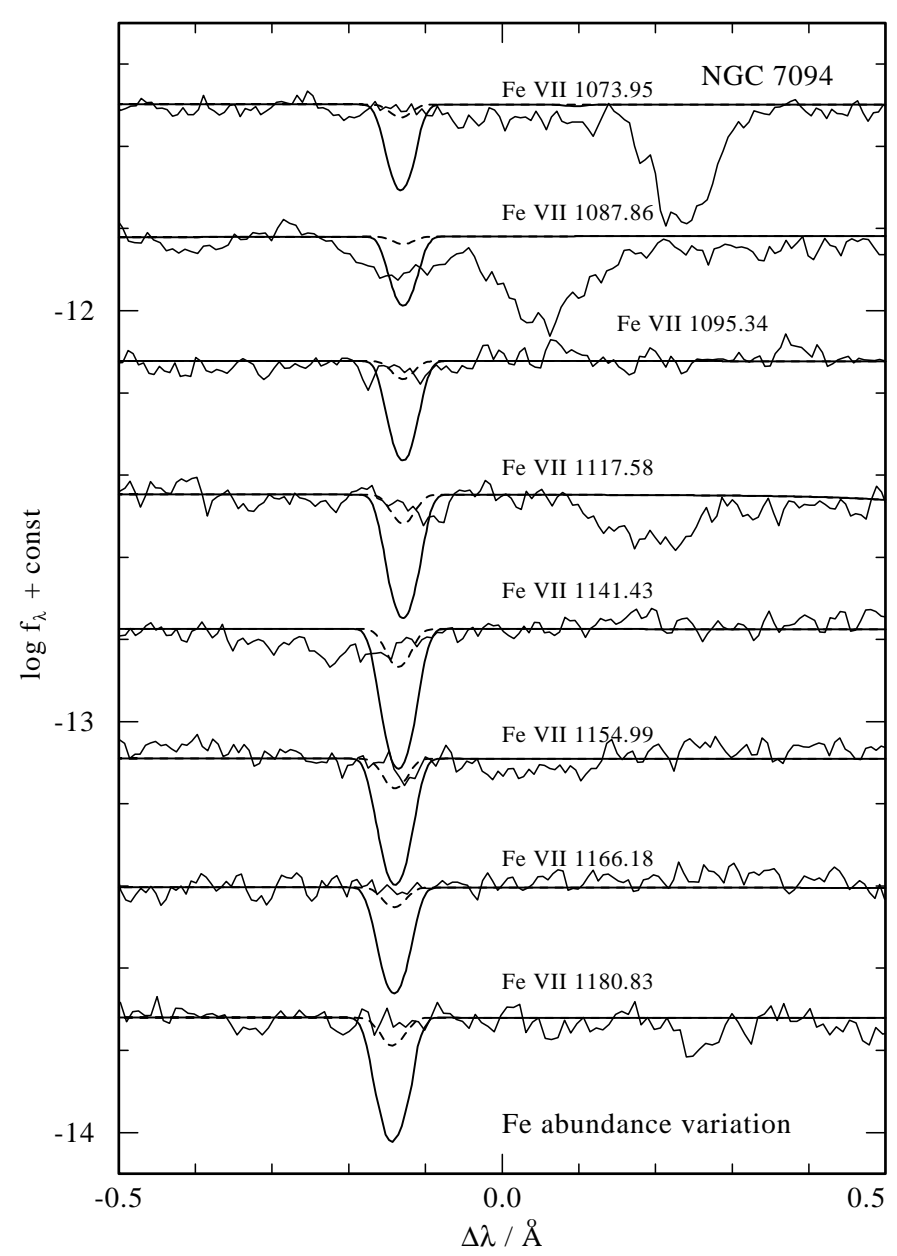

Fig. 6. Details of FUSE spectrum of NGC 7094 compared to theoretical line profiles, centered around the strongest Fe VII lines in the model. The model iron abundances are 0.1 solar and 0.01 solar. No iron lines are detectable in the FUSE spectrum. Spectra are smoothed with $0.01 \AA$ Gaussians.

\subsection{DO white dwarfs}

KPD 0005+5106 is the hottest known DO white dwarf, showing peculiar emission lines due to ultrahigh ionized light metals (Werner \& Heber 1992) and it is the only white dwarf known to have an X-ray corona (Fleming et al. 1993). An upper limit for the Fe abundance can be inferred from the GHRS spectrum (one tenth solar, Fig. 7). As in the case of RX J 2117.1+3412, the absorption feature near $1240 \AA$ cannot stem from Fe VII, because no other Fe VII lines, neither in the HST nor in the FUSE data, are detectable. We derive an upper limit of $\log (\mathrm{Fe} / \mathrm{He})<-5$ (by number).

PG 1034+001 is another hot DO white dwarf for which an iron abundance of $\log (\mathrm{Fe} / \mathrm{He})=-5$ has been derived from Fe vi lines in HST data (Werner et al. 1995). We confirm this result by our re-analysis.

HZ 21 is one of the coolest DO white dwarfs. We confirm the result by Holberg et al. (1998) that only a single photospheric line can be identified in the IUE spectra (He II $1640 \AA$ ). The HST spectra are superior in quality, but they only cover wavelength ranges where Fe VI and 


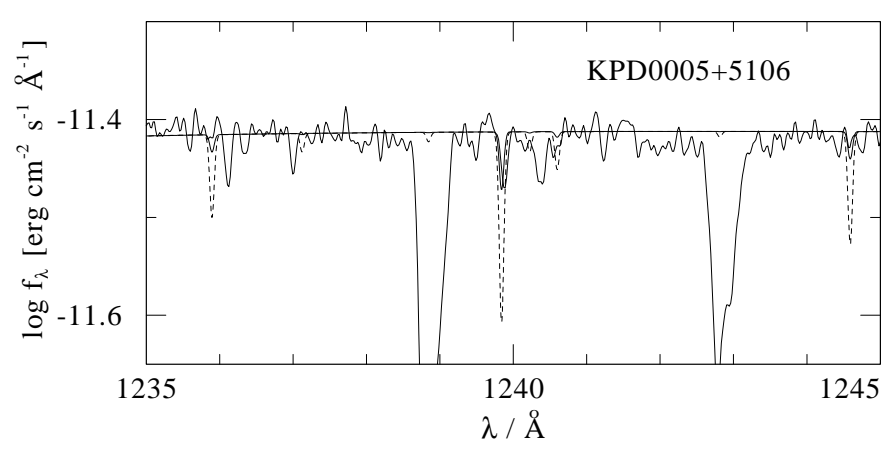

Fig. 7. The strongest Fe VII lines expected in the HST spectrum of KPD $0005+5106$. We show models with an iron abundance of 0.1 solar (solid line) and solar (dashed line). No Fe lines can be identified. The feature at $1240 \AA$ is probably interstellar Mg II. The HST spectrum is smoothed with $0.06 \AA$ Gaussian. A radial velocity shift of $+36.15 \mathrm{~km} \mathrm{~s}^{-1}$ is taken into account (Holberg et al. 1998).

Fe VII are located. HZ 21 is too cool to ionize iron this strongly. We derive an upper limit of $\log (\mathrm{Fe} / \mathrm{He})<-4$.

HD149499B is also a cool DO star. From the absence of $\mathrm{Fe} \mathrm{V}$ lines in the HST spectrum we derive an upper limit for the Fe abundance: $\log (\mathrm{Fe} / \mathrm{He})<-5$.

To conclude our results on the DO stars, the iron abundance is much lower than predicted by diffusion theory (Fig. 8), confirming earlier results on other DO white dwarfs (Dreizler 1999).

\subsection{Summary of iron line analyses}

To summarize this section, we fail to identify iron lines in any PG 1159 star. In most objects this is compatible with a solar iron abundance. In three cases (PG 1159035, HS 2324+397, PG $1520+525)$ we tentatively propose an interpretation in terms of a slight iron underabundance (0.5 dex). In three cases (K 1-16, NGC 7094, Abell 78) the underabundance is estimated to 1-2 dex.

For three out of four DO white dwarfs investigated in this work we derive an upper iron abundance limit from the absence of respective lines in their spectra. In the case of PG $1034+001$ the analysis of detected iron lines corroborates an earlier abundance determination.

\section{Detection of sulfur in K 1-16 and other PG 1159 stars}

The FUSE spectrum of K1-16 allows for the first identification of sulfur in a PG 1159 star based on the 933.38/944.52 $\AA$ resonance doublet (Fig. 9). It seems that both lines are split into two components, especially the S vi $933.38 \AA$ line. The stronger blueshifted components are attributed to the photosphere. Their velocity shift is about $-40 \mathrm{~km} \mathrm{~s}^{-1}$ which is in coarse agreement with measurements from IUE spectra $\left(-48.07 \mathrm{~km} \mathrm{~s}^{-1}\right.$, Holberg et al. 1998). Our model fit suggests a solar sulfur abundance.

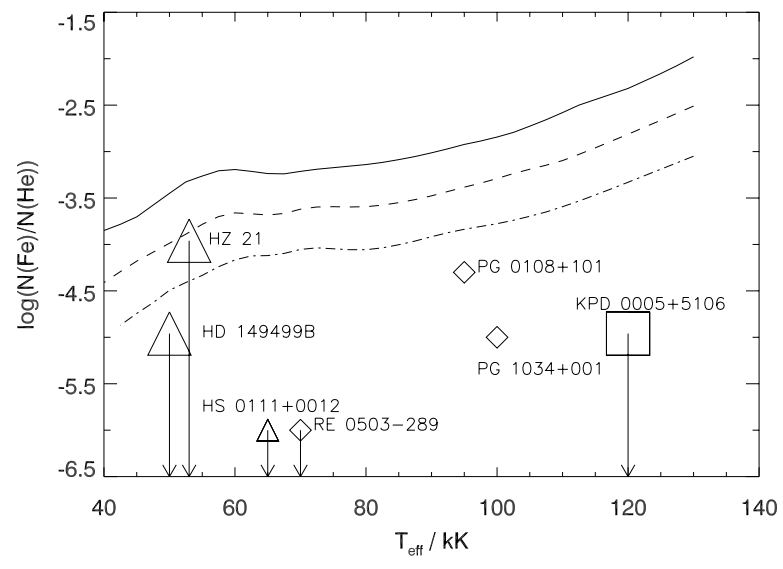

Fig. 8. Comparison of predicted iron abundances to our results for the DO white dwarfs. The graphs show diffusion equilibrium abundances for iron at $\tau=2 / 3$ as calculated by Chayer etal. (1995): $\log g=7.0$ (solid line), $\log g=7.5$ (dashed), $\log g=8.0$ (dash-dotted). The symbols mark objects with $\log g=7.0(\square), \log g=7.5(\diamond)$ and $\log g=8.0(\triangle)$. Small symbols mark the objects analyzed by Dreizler (1999).

The $\mathrm{S}$ VI doublet is also detected in the spectra of RX J 2117.1+3412, PG 1520+525, and PG 1159-035.

\section{Discussion}

Whether the iron deficiency in PG 1159 stars is related to the same phenomenon observed in metal-poor postAGB B-A-F supergiants can only be speculated upon. In some cases dust fractionation on the AGB has been invoked to explain the Fe deficiency (Van Winckel et al. 1992), whereas in other cases evidence was found that iron was transformed to heavier elements by s-process neutron captures (Decin et al. 1998). To our opinion it is unlikely that dust fractionation on the AGB is responsible for the iron deficiency in PG 1159 stars, because strong post-AGB mass-loss removes layers "cleaned" from iron during the AGB phase.

In the case of PG 1159 stars the high C and O abundances result from envelope mixing caused by a late Heshell flash (Herwig et al. 1999). This event also modifies the near-solar abundance ratios of iron-peak elements in the envelope by dredging up matter in which $s$-process elements were built-up by $n$-capture on ${ }^{56} \mathrm{Fe}$ seeds during the AGB phase. This scenario can be tested by analyzing the resulting $\mathrm{Fe} / \mathrm{Ni}$ abundance ratio, because it is significantly changed in the intershell region in favor of $\mathrm{Ni}$ by the conversion of ${ }^{56} \mathrm{Fe}$ into ${ }^{60} \mathrm{Ni}$. The Fe depletion by $n$-captures typically amounts to a factor of 10 (Busso et al. 1999). In order to roughly estimate the $\mathrm{Ni} / \mathrm{Fe}$ ratio one can assume nuclear statistical equilibrium. The two most abundant $\mathrm{Ni}$ isotopes are ${ }^{60} \mathrm{Ni}(26 \%)$ and ${ }^{58} \mathrm{Ni}(68 \%)$. During s-process ${ }^{58} \mathrm{Ni}$ is destroyed (and not synthesized), by conversion into ${ }^{60} \mathrm{Ni} .{ }^{60} \mathrm{Ni}$ is converted to heavier elements 4 times faster than it is produced from ${ }^{56} \mathrm{Fe}$. Consequently, 


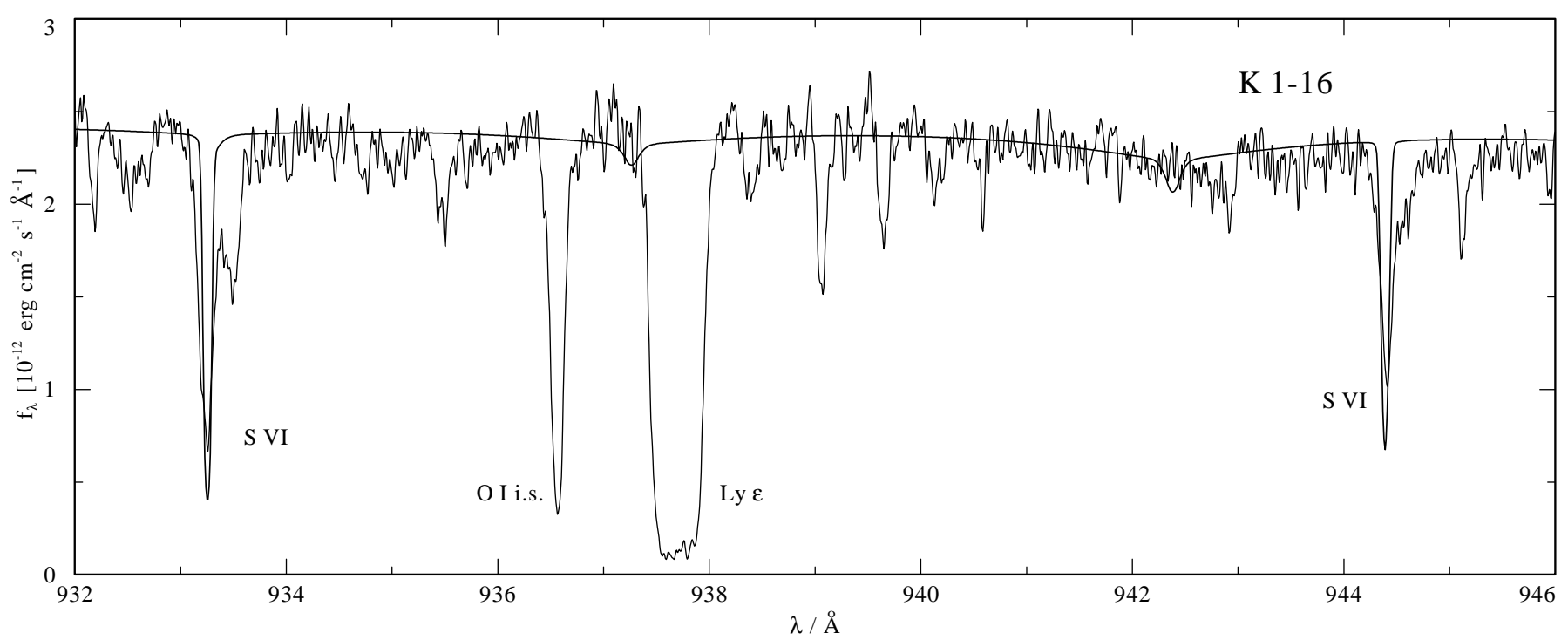

Fig. 9. Line profile fit to the S VI resonance doublet in the FUSE spectrum of K 1-16. The model has a solar sulfur abundance. Other parameters as in Table 1 . The spectra are smoothed with $0.02 \AA$ Gaussians.

a ratio $\mathrm{Fe} / \mathrm{Ni} \approx 4$ results, which is a factor of five below the solar value. This could be detected by high resolution HST and FUSE UV spectroscopy of PG 1159 stars, but only in objects that are cooler than the three hot stars for which the strong Fe deficiency has been found. This is because in the hot PG 1159 stars Ni VII lines are the prevailing nickel features but they are located in the inaccessible EUV region. Interestingly, Asplund et al. (1999) have indeed found that in Sakurai's object, which is thought to undergo a late He-shell flash, Fe is reduced to 0.1 solar and $\mathrm{Fe} / \mathrm{Ni} \approx 3$. This and other $s$-process signatures might also be exhibited by Wolf-Rayet central stars and PG 1159 stars.

More quantitative results from nucleosynthesis calculations in appropriate stellar models have been presented recently (Herwig et al. 2002) and inclusion of nuclear networks in evolutionary model sequences will become available in the near future.

Most recent results presented at the IAU Symposium 209 (Planetary Nebulae, Canberra) confirm that iron deficiency among H-deficient post-AGB stars is not restricted to PG 1159 stars, as can be expected from evolutionary considerations. As already mentioned, the [WC]-PG 1159 transition object Abell 78 is iron deficient (Werner et al. 2002) and three Wolf-Rayet central stars are iron deficient, too. Gräfener et al. (2002) report a low $\mathrm{Fe}$ abundance in SMP 61, an early type [WC5] central star in the LMC. Its abundance is at least 0.7 dex below the LMC metallicity. Crowther et al. (2002) find evidence for an iron underabundance of $0.3-0.7$ dex in the Galactic [WC] stars NGC 40 ([WC8]) and BD+303639 ([WC9]).

A general iron deficiency among PG 1159 stars would also have implications for asteroseismology. Roughly half of all PG 1159 stars are GWVir pulsators and frequency analyses have revealed interesting results about the interior structure of these stars (e.g. Winget et al. 1991). There is still a debate about details of the pulsation driving mechanism. While it is accepted that cyclic ionization of carbon and oxygen just beneath the photosphere is the main driver, the iron opacity also does play a role (Saio 1996). In light of our iron abundance analysis of the pulsator K1-16 it would be interesting to re-address the problem of pulsation driving.

Acknowledgements. We thank Falk Herwig for useful discussions. HST data analysis in Tübingen is supported by the DLR under grant 50 OR 97055 . JLD is supported by the DFG under grant We 1312/23-1.

\section{References}

Asplund, M., Lambert, D. L., Kipper, T., Pollacco, D., \& Shetrone, M. D. 1999, A\&A, 343, 507

Bashkin, S., \& Stoner, J. O. Jr. 1975, Atomic Energy Levels And Grotrian Diagrams (North-Holland/American Elsevier)

Busso, M., Gallino, R., \& Wasserburg, G. J. 1999, ARA\&A, 37,239

Chayer, P., Fontaine, G., \& Wesemael, F. 1995, ApJS, 99, 189

Crowther, P. A., Abbott, J. B., Hillier, D. J., \& De Marco, O. 2002, in Planetary Nebulae, ed. M. Dopita et al., IAU Symp., 209, ASP Conf. Ser., in press

Decin, L., Van Winckel, H., Waelkens, C., \& Bakker, E. J. 1998, A\&A, 332, 982

Deetjen, J. L., Dreizler, S., Rauch, T., \& Werner, K. 1999, A\&A, 348, 940

Dreizler, S. 1999, A\&A, 352, 632

Dreizler, S., \& Werner, K. 1996, A\&A, 314, 217

Dreizler, S., \& Heber, U. 1998, A\&A, 334, 618

Dreizler, S., Werner, K., Heber, U., \& Engels, D. 1996, A\&A, 309, 820

Dreizler, S., Werner, K., \& Heber, U. 1997, in Planetary Nebulae, ed. H. J. Habing, \& H. J. G. L. M. Lamers, IAU Symp., 180 (Kluwer), 103 
Feibelman, W. A. 1995, PASP, 107, 531

Feibelman, W. A. 1999, ApJ, 513, 947

Feibelman, W. A. 2000, ApJ, 542, 957

Feibelman, W. A., \& Bruhweiler, F. C. 1990, ApJ, 357, 548

Feibelman, W. A., \& Johansson, S. 1995, ApJS, 100, 405

Fleming, T. A., Werner, K., \& Barstow, M. A. 1993, ApJ, 416, L79

Gräfener, G., Hamann, W.-R., \& Peña, M. 2002, in Planetary Nebulae, ed. M. Dopita et al., IAU Symp., 209, ASP Conf. Ser., in press

Herwig, F., Blöcker, T., Langer, N., \& Driebe, T. 1999, A\&A, 349, L5

Herwig, F., Lugaro, M., \& Werner, K. 2002, in Planetary Nebulae, ed. M. Dopita et al., IAU Symp., 209, ASP Conf. Ser., in press

Holberg, J. B., Barstow, M. A., \& Sion, E. M. 1998, ApJS, 119, 207

Kruk, J. W., \& Werner, K. 1998, ApJ, 502, 858

Kurucz, R. L. 1991, in Stellar Atmospheres: Beyond Classical Models, ed. L. Crivellari, I. Hubeny, \& D. G. Hummer, NATO ASI Ser. C, 341, 441

Moos, H. W., Cash, W. C., Cowie, L. L., et al. 2000, ApJ, 538, L1

Napiwotzki, R., Hurwitz, M., Jordan, S., etal. 1995, A\&A, 300, L5

Rauch, T., \& Werner, K. 1997, in The Third Conference on Faint Blue Stars, ed. A. G. D. Philip, J. Liebert, \& R. A. Saffer, (L. Davis Press, Schenectady, NY), 217
Sahnow, D. J., Moos, H. W., \& Ake, T. B. 2000, ApJ, 538, L7

Saio, H. 1996, in Hydrogen-Deficient Stars, ed. U. Heber, \& C. S. Jeffery, ASP Conf. Ser., 96, 361

Seaton, M. J., Yu, Yan, Mihalas, D., \& Pradhan, A. K. 1994, MNRAS, 266, 805

Van Winckel, H., Mathis, J. S., \& Waelkens, C. 1992, Nature, 356,500

Werner, K. 1996, A\&A, 309, 861

Werner, K. 2001, in Low mass Wolf-Rayet stars: Origin and evolution, ed. T. Blöcker, L. B. F. M. Waters, \& A. A. Zijlstra, Ap\&SS, 275, 27

Werner, K., \& Heber, U. 1992, in Atmospheres of Early-Type Stars, ed. U. Heber, C. S. Jeffery (Springer, Berlin), Lect. Notes Phys., 401, 291

Werner, K., \& Koesterke, L. 1992, in Atmospheres of EarlyType Stars, ed. U. Heber, \& C. S. Jeffery (Springer, Berlin), Lect. Notes Phys., 401, 288

Werner, K., \& Dreizler, S. 1999, in Computational Astrophysics, ed. H. Riffert, \& K. Werner, J. Comp. Appl. Math., 109, 65

Werner, K., Heber, U., \& Hunger, K. 1991, A\&A, 244, 437

Werner, K., Dreizler, S., \& Wolff, B. 1995 A\&A, 298, 567

Werner, K., Dreizler, S., Heber, U., et al. 1996, A\&A, 307, 860

Werner, K., Dreizler, S., Koesterke, L., \& Kruk, J. W. 2002, in Planetary Nebulae, ed. M. Dopita et al., IAU Symp., 209, ASP Conf. Ser., in press

Winget, D. E., Nather, R. E., Clemens, J. C., et al. 1991, ApJ, 378,326 